Pré-Publicações do Departamento de Matemática

Universidade de Coimbra

Preprint Number 14-41

\title{
AUTOMATED RETINA IDENTIFICATION BASED ON MULTISCALE ELASTIC REGISTRATION
}

\author{
ISABEL NARRA FIGUEIREDO, SUNIL KUMAR, JÚLIO S. NEVES, SUSANA MOURA, \\ CARLOS MANTA OLIVEIRA AND JOÃO DIOGO RAMOS
}

\begin{abstract}
In this work we propose a novel method for identifying individuals based on retinal fundus image matching. It consists in extracting the retina vasculatures from the images, and subsequently in registering the vessel networks by using a non-parametric approach, that involves a multiscale elastic registration procedure. Afterwards a decision identification function, relying on a suitable normalized function, is defined to decide whether or not the pair of images belongs to a particular individual. The method is tested on a data set of 21680 pairs generated from a total of 946 images of 339 different individuals. It achieves $98.830 \%$ of accuracy, $91.350 \%$ of sensitivity and $99.998 \%$ of specificity, which shows the reliability of the method.
\end{abstract}

KEYWORDS: Retinal fundus images. Elastic image registration. Vessel network.

\section{Introduction}

Biometrics has quickly established itself as the most pertinent technology for identifying individuals in a fast and reliable way through the use of unique biological characteristics. Biometric identification is stronger than traditional passwords because it cannot be compromised by guessing or through the use of brute-force computing power. Up until fairly recently, biometrics was used mostly in high-security applications such as law enforcement, passports and access to military bases. The popularly used biological characteristics for identification purposes are face, fingerprint, hand geometry, retina, iris, vein, voice, and so on [19]. Among these, the retina can provide a higher level of security due to the fact that each eye has its own totally unique pattern of blood vessels [3]. Even genetically identical twins were found to have different pattern of retinal blood vessels [21]. Moreover, with the emergency of EHR (Electronic Health Records) and as equipments are becoming increasingly affordable, the automatic assessment of who a retinal picture belongs to, allows for automation of processes and reduction of errors.

The first method on retina identification dates back to 1976 when EyeDentify company introduced the system named EyeDentification 7.5 [12].

Received November 24, 2014. 
Since then several new methods have been reported in the literature, see for instance $[1,16,6,13,18,17,7]$ and the references therein. The method proposed in [13] was based on the Scale Invariant Feature Transform (SIFT) and the Improved Circular Gabor Transform (ICGT). The method in [6] used Harris corner detector for feature extraction and phase correlation technique to estimate the rotation angle of head or eye movement in front of a retina fundus camera. Then, a new similarity function was proposed to compute the similarity between features of different retina images. The authors in [16] first defined a set of feature points using the ridge endings and ridge bifurcations from the retinal vessels, and then registered them to measure the degree of similarity between the input images. In [1] the blood vessels pattern are extracted from the retina images, and morphological thinning process is applied on the extracted pattern. After thinning, two feature vectors based on the angular and radial partitioning of the pattern image are defined. Then the feature vectors are analyzed using 1D discrete Fourier transform, and the Manhattan metric is used to measure the closeness of the feature vectors. The authors in [7] proposed a novel method based on the features obtained from retinal images. The method was composed of three principal modules including blood vessel segmentation, feature generation, and feature matching. The authors in [18] extracted the features based on Fourier transform and angular partitioning of the spectrum, and employed the Euclidean distance for feature matching.

In this work we propose a novel identification method using retinal fundus images. First of all, we extract the retina vasculatures from the images (as demonstrated in $[19,21]$, retina blood vessel's pattern is unique among individuals and forms a good differentiation between them). Since the vessels appear most contrasted in the green channel we use it for extracting the vessels, first by denoising it, applying a fast discret curvelet transform [5] and then using the isotropic undecimated wavelet transform [20]. Secondly, we register the extracted retina vasculatures using a multiscale elastic registration strategy with an affine pre-registration, based on $[14,15]$. Finally, we define appropriate decision functions, or equivalently similarity measures, relying on suitable normalized functions. They are devised to serve as binary classifiers for deciding, after the registration process, whether a pair of retinal fundus images belongs or not to the same individual (meaning the same individual eye). The method is tested on a data set of 21680 image pairs generated from 946 images of 339 different individuals. The images 
are provided by the company Retmarker (http://www.retmarker.com/), and are taken during an ongoing screening program in Portugal. The proposed method achieves $99.998 \%$ of specificity, $91.350 \%$ of sensitivity and $98.830 \%$ of accuracy.

The layout of the paper is as follows. The elastic image registration model is revised in Section 2, and some comments about the appropriateness to use it in this retina identification context are presented. The proposed methodology (including the decision function), for identifying individuals based on retinal fundus images, as well as a summary of the algorithm, are explained in Section 3. The evaluation of the method on a large data set is done is Section 4. The last section closes the paper with some conclusions and outlook.

\section{Elastic image registration}

In a general sense, image registration is a method in image processing that consists in comparing the inbuilt information carried by different images of the same object (or scene), taken at different times, and/or by different devices. It has many different applications, as for example, i) medical image analysis (as monitoring tumor growth, quantifying disease progression over time in a patient, comparison with anatomical atlases), ii) biometrics (for identification, which is the process of presenting an identity to a system, and for recognition/authentication, which is the process of validating or proving an identity provided to a system) or iii) remote sensing (for environmental monitoring, change detection, weather forecasting).

More exactly, given a pair of images, one called the reference $R$ (and that is kept unchanged) and the other called the template $T$, the goal is to find a geometric transformation $\varphi$, mapping points from the template image $T$ onto the reference image $R$, in such a way that the transformed template image, denoted by $T(\varphi)$, becomes similar to the reference image $R$.

There are two main classes of image registration models depending of the type of the geometric transformation : parametric image registration (if the transformation is parametric, i.e, it can be represented in terms of some parameters and basis functions), or non-parametric image registration (when the transformation is a more general function).

The image registration approach we propose in this paper relies on the so-called elastic registration model, cf. [4, 14], described in Section 2.1, and that is a non-parametric image registration model. 
2.1. Optimization model. Let the given reference and template images be represented by the functions $R, T: \Omega \subset \mathbb{R}^{2} \longrightarrow \mathbb{R}$, where $\Omega$ stands for the pixel domain, of size $n_{1} \times n_{2}$. For each pixel $x=\left(x_{1}, x_{2}\right)$ in $\Omega$, we denote by $\varphi:=\left(\varphi_{1}, \varphi_{2}\right): \Omega \subset \mathbb{R}^{2} \longrightarrow \mathbb{R}^{2}$ the unknown coordinate transformation that originates the desired alignment between the reference image $R$ and the transformed template image, denoted by $T(\varphi)$. Furthermore for the partial derivatives with respect to $x_{1}$ and $x_{2}$ we use the notations $\partial_{1}$ and $\partial_{2}$, respectively. Hereafter we also split $\varphi$ into the trivial identity part and the deformation or displacement part $u$, which means,

$$
\varphi(x):=x-u(x), \quad \text { with } \quad u:=\left(u_{1}, u_{2}\right),
$$

and introduce

$$
T(u(x)):=T(x-u(x)) .
$$

The elastic image registration model is an optimization problem composed of three main parts, that are shortly explained here in a continuous setting :

- The similarity measure $\mathcal{D}$ (also called the distance measure or the misfit term) that quantifies the similarity or de-similarity of the reference and template images, under the transformation $u$. The chosen $\mathcal{D}$ is the sum of squared differences (SSD)

$$
\begin{aligned}
\mathcal{D}(R, T(u)) & :=\frac{1}{2}\|T(u)-R\|_{L^{2}(\Omega)}^{2} \\
& =\frac{1}{2} \int_{\Omega}(T(x-u(x))-R(x))^{2} d x
\end{aligned}
$$

where $L^{2}(\Omega)$ is the space of square-integrable functions in $\Omega$.

- A regularization term denoted by $\mathcal{S}$ (which should make the optimization problem well-posed) and whose goal is to rule out non-reasonable solutions, or in other words, to restrict the transformation $u$ to an appropriate type. The chosen $\mathcal{S}$ is the elastic potential that measures the energy introduced by deforming an elastic material. It is defined by

$$
\mathcal{S}(u):=\int_{\Omega}\left(\frac{\lambda+\mu}{2}\|\operatorname{div} u\|^{2}+\frac{\mu}{2} \sum_{i=1}^{2}\left\|\nabla u_{i}\right\|^{2}\right) d x .
$$

Here $\nabla$ and div denote, respectively, the gradient and divergence operators

$$
\nabla u_{i}:=\left(\partial_{1} u_{i}, \partial_{2} u_{i}\right), \quad \text { div } u:=\partial_{1} u_{1}+\partial_{2} u_{2}, \quad(\text { for } i=1,2),
$$


$\|$.$\| is the notation for the Euclidean norm, and the parameters \lambda$ and $\mu$ are the Lamé constants characterizing the elastic material.

- The objective functional is then defined by

$$
\mathcal{J}(u):=\mathcal{D}(R, T(u))+\alpha \mathcal{S}(u),
$$

and finally the optimization model is

$$
\min _{u} \mathcal{J}(u)
$$

where $\alpha>0$ is a regularization parameter that balances the influence of the similarity and regularity terms in the cost functional $\mathcal{J}$.

We comment now on the several components that constitute this elastic registration model and its suitability for retina identification, as proposed in this paper. Concerning the similarity (or distance) measure, many other measures exist in the literature (as for instance, cross-correlation, mutual information, normalized gradient fields), and the nontrivial and difficult question which one should be used for a particular application arises. One of the simplest measures, for mono-modal registration, is the SSD defined in (1), that is also appropriate for optimization. Essentially, the SSD measure matches intensity patterns over the whole image. As explained in the following Section 3, the registration approach, we propose herein, is a procedure that relies on the registration of the retinal vasculature binary images (that embody crucial, unique and identifiable anatomical features of the retina), which are appropriately interpolated at different scales, for performing a multiscale elastic registration. Therefore the SSD measure, when applied to the vasculature images, becomes an hybrid measure that combines geometric and intensity features. As demonstrated in Section 4, the choice of this distance measure leads to very good registration results.

Regarding the choice of the deformation $u$ (that defines how one image can deform to match another), other possibilities exist (for instance other type of non-parametric transformation, or a parametrized deformation). But it is well known that the majority of the human body organs and structures are made of soft tissues, therefore the choice of an elastic deformation seems very appropriate for the retinal fundus images, to handle small deformations of soft tissues during imaging.

By computing the Gâteaux derivative of the objective functional $\mathcal{J}$, the elastic deformation $u$, which is a minimizer of $\mathcal{J}$, can be characterized by the 
following PDEs (partial differential equations),

$$
\mu \Delta u+(\lambda+\mu) \nabla \operatorname{div} u=\frac{1}{\alpha}\left(R-T_{u}\right) \nabla T_{u} .
$$

(where $T_{u}$ is the derivative of $T$ with respect to $u$ ) and that are nothing more than the Navier-Lamé equations. These PDEs show that in the elastic registration model (5), the two images can be understood as an undeformed (reference image) and a deformed (template image) elastic two-dimensional body, that obey to the linear elasticity theory. Following the equation (6), the template image is deformed by the action of the external forces (the right hand side of (6)) generated by the image similarity measure, until the internal forces of the body (the left hand side of (6)) and the external forces (defined by the image matching mis-fit) reach the equilibrium. We remark that the linear elasticity assumption is only valid for small deformations, so for registering large image differences another transformation, as e.g. an affine transformation, is necessary to overcome this problem, as explained in Section 3.2.

In general an analytical solution to (5) does not exist, and consequently the optimization problem (5) is then discretized and gives rise to a finite dimensional problem. The numerical scheme used in this paper to solve the discretized version of (5) is a Gauss-Newton like method (with Armijo's line search).

We finish this section with some comments on the choice of the parameters $(\mu, \lambda$ and $\alpha)$ involved in the definition of the elastic registration model (5).

The optimal value for the regularization parameter $\alpha$ is not known. A continuation method in $\alpha$, as suggested in $[10,11]$, can be used to determine its value. It consists in starting with a large $\alpha_{0}$, and to compute a sequence of solutions $u^{\alpha_{j}}$ of $\min _{u}\left(\mathcal{D}(R, T(u))+\alpha_{j} \mathcal{S}(u)\right)$, with $\alpha_{j+1}<\alpha_{j}$, and to use $u^{\alpha_{j}}$ as the starting guess for the solution of the next registration problem for $\alpha_{j+1}$. This method ends when $\mathcal{D}\left(R, T\left(u^{\alpha_{j}}\right)\right)<c$, where $c$ is a given parameter, monitored by a trained professional. The optimal value we have used is $\alpha=800$ (see Section 4).

Regarding the Lamé parameters, $\mu$ and $\lambda$, it is well known that, for example, increasing values of $\mu$ mean a more rigid material, $\lambda=0$ means that the body shows no contraction under deformation. In our experiments performed in a large dataset (see Section 4), we have always considered $\lambda=0$, which is a common choice in several medical elastic image registration problems (cf., 
e.g, [4]) and regarding $\mu$ we have tested the values $\mu=1,2,3,4,5,9,10,15,20$. The best results were obtained for $\mu=9$ (see Section 4).

\section{Registration approach}

Our approach is a multiscale elastic image registration (based on $[14,15]$ ) of the retina vasculature (with a pre-registration step). In effect, the retinal vascular network is itself a unique structure to each individual. The awareness of this fact was first stated in [19], where it is reported that every retina contains a unique blood vessel pattern. Subsequently in [21] it is conducted a study, that concludes that even among identical twins the blood vessel patterns are different and unique.

In this section firstly we explain how the vasculature is extracted. Secondly we describe the pre-registration process, that is used as the starting point in the multiscale elastic registration process. Finally we define the function devised to serve as binary classifier for deciding, after the registration process, whether a pair of retinal fundus images belongs or not to the same individual (meaning the same individual eye).

3.1. Vessel network extraction. In retinal fundus images the vessels appear most contrasted in the green channel compared to the red and blue channels of the RGB image. To improve the quality of the vessel extraction we firstly perform a pre-processing step previous to vasculature segmentation, by denoising the green channel using fast discrete curvelet transforms [5]. This intends to ameliorate the subsequent retinal identification procedure. As explained in [5] "a curvelet transform is a multiscale pyramid with many directions and positions at each length scale and needle-shaped elements at fine scales". In particular curvelets are appropriate for representing twodimensional images exhibiting objects with edges, i.e., curved line segments, as for instance vessels. Figure 1 displays two retinal fundus images (subfigures (a) and (c)), the corresponding green channels (subfigures (b) and (d)) and the denoised green channels (second row) using different percentages of coefficients (1\% and 10\%) in the partial curvelet reconstruction of the green channels. To obtain these denoised green channels we have applied the fast discret curvelet transform via wrapping, as implemented in CurveLab available at http://www.curvelet.org. (the wrapping version uses a decimated rectangular grid aligned with the image axes, to translate curvelets at each scale and angle, see [5]). 


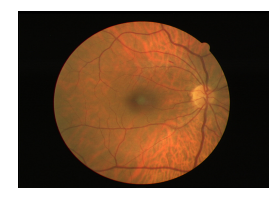

(a)

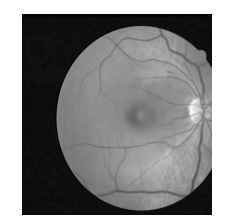

(e)

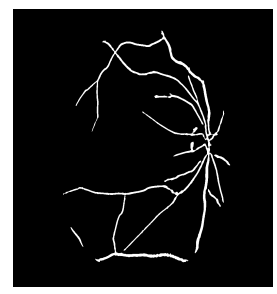

(i)

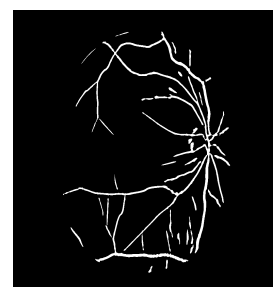

$(\mathrm{m})$

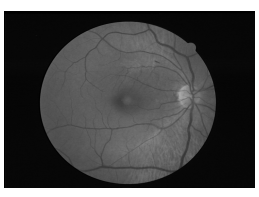

(b)

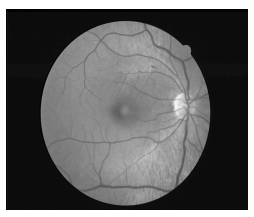

(f)

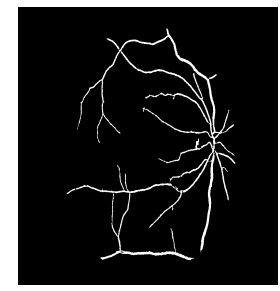

(j)

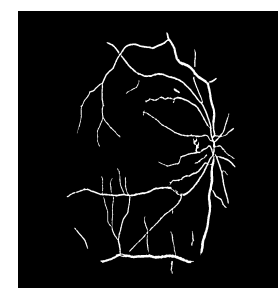

(n)

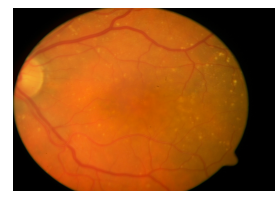

(c)

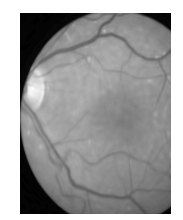

(g)

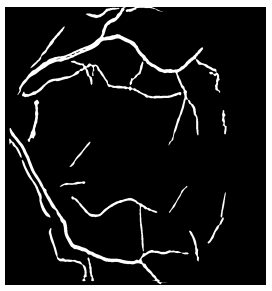

(k)

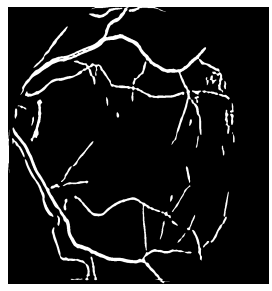

(o)

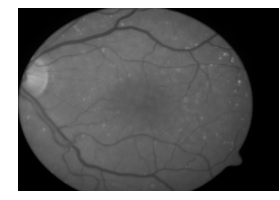

(d)

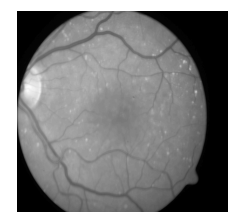

(h)

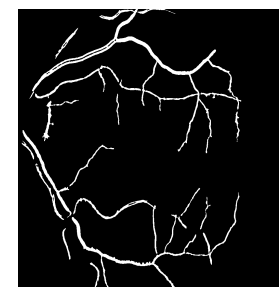

(1)

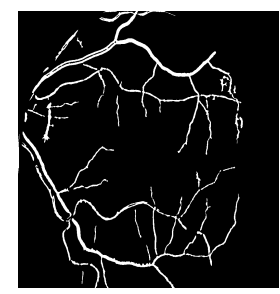

(p)

Figure 1. First and third columns : (a) and (c) original retinal fundus images; (e) and (g) denoised green channels with a percentage equal to $1 \%$ of coefficients used in the partial curvelet reconstruction; (i), (k) and (m), (o) binary vasculature images by removing objects of size less than 500 and 150 pixels. Second and fourth columns : (b) and (d) green channels of images (a) and (c), respectively; (f) and (h) denoised green channels with a percentage equal to $10 \%$ of coefficients used in the partial curvelet reconstruction; (j), (l) and (n), (p) binary vasculature images by removing objects of size less than 500 and 150 pixels, respectively. 
The vessel network is then obtained, from the denoised green channel, using the isotropic undecimated wavelet transform [20] and by employing an approach similar to [2]. Briefly this approach includes the following steps i) to iv) : i) Retinal fundus images normally contain a main region at the center of the image, which is called the field of view (FOV), surrounded by dark background pixels. For extracting the vessels we only need to process the main region, so we separate it from the background, in a preprocessing step, and this separation is performed by eroding the green channel of the image, using a square structuring element. ii) We compute the sum of the second and third wavelet levels of the denoised green channel of the image and extract the darkest $10 \%$ pixels within the FOV. iii) The vasculature can be seen as a large connected structure in the binary image, along with some isolated small objects, which are removed from the binary image. In a similar way we fill small holes present within the thresholded regions. iv) We remove objects of size less than 500 pixels and fill holes of size greater than 20 pixels.

In Figure 1, the eight subfigures (i) to (p) show binary images corresponding to the vessel networks of the fundus images using $1 \%$ and $10 \%$ of the coefficients in the curvelets (see second row) and by removing of objects of size less than 500 pixels (third row) and also size less than 150 pixels (fourth row).

As expected, it can be easily observed, that by using a big percentage of coefficients and keeping objects of size small more details are captured. However, we have decided to use only " $1 \%$ of the coefficients" and "to remove objects of size less than 500 pixels ", for speeding up the procedure and because the vasculature is a large connected structure, respectively. In addition these two choices have demonstrated to perform quite well in the large dataset we have used, as explained in Section 4.

3.2. Pre-registration. The pre-registration step used corresponds to a parametric image registration method. It can be shortly defined in the following way: it is again an optimization problem like (4)-(5), where the the cost functional in (4) does not have the regularizing term, the misfit function is again the sum of squared differences, but the transformation $u$ is now an affine two-dimensional transformation. It can be characterized by six parameters, that is, $u=\left(u_{1}, u_{2}\right)$, where $u_{1}:=w_{1} x_{1}+w_{2} x_{2}+w_{3}$ and $u_{2}:=w_{4} x_{1}+w_{5} x_{2}+w_{6}$. The corresponding optimization problem is solved by a Gauss-Newton type method. 
We observe that affine transformation is the most common used method in registering two images. Although only linear, it involves four simple transformations (translation, rotation, scale and shear) and consequently it can correct some main distortion. On the other hand, the linear elastic transformation (described in Section 2, formula (2)) is invariant to rigid transformations (this latter is a composition of translations and rotations). Therefore a pre-registration step with an affine transformation can be an advantageous pre-step to the overall proposed registration procedure.

3.3. Multiscale elastic image registration. The multiscale approach corresponds to a multiscale representation of the data and consequently leads to a sequence of optimization problems of the form (4)-(5). It is a strategy that attempts to diminish or eliminate several possible local minima and lead to convex optimization problems. To be precise, let $\theta \in \mathbb{R}$ denote a scale parameter, associated to a spline interpolation procedure [15]. By starting with a large initial $\theta$, the corresponding interpolated reference and template images, $R(\theta)$ and $T(\theta)$, will retain only the most prominent features (small details in these images will disappear). Therefore a numerical solution $u(\theta)$, of the elastic image registration problem (4)-(5) for the input images $R(\theta)$ and $T(\theta)$, is computed at this coarse scale where the optimization scheme is less likely to be trapped in local minima (because the image interpolation at the coarse scale intends to get rid of these local minima). The solution $u(\theta)$ is used afterwards as a starting point for the elastic image registration at the following finer scale, aiming at speeding up the total optimization procedure.

Figures 2 and 3 show the result of the global registration process for two images of the same subject eye, using FAIR software [15]. In particular in Figure 2, the results for the final transformed template vasculature $T(u)$, for a single scale $\theta=100$, for two scales $\theta=\left[\begin{array}{lll}100 & 10\end{array}\right]$, for three scales $\theta=\left[\begin{array}{lll}100 & 10 & 1\end{array}\right]$ and finally for four scales $\theta=\left[\begin{array}{llll}10 & 10 & 1 & 0\end{array}\right]$ are exhibited. In Figure 4 the final transformed templates, corresponding to two different pairs of images are displayed (one pair is made of images of the right eye of the same subject eye and the other pair is made of images of the left eye of different subjects).

The performance of each one of these decision functions, for the large data set used, was quite similar, but $d_{1}$ has proven to be a little bit superior than the others (see Section 4 for the results concerning $d_{1}$ ). 


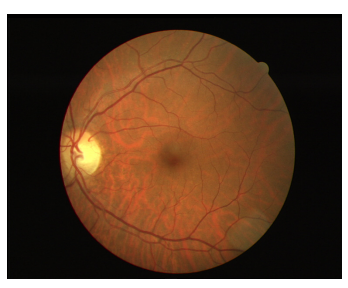

(a)

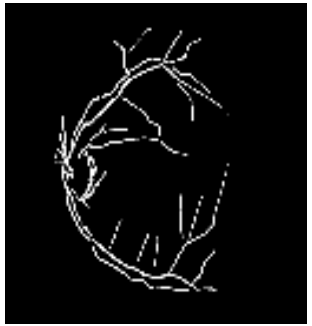

(c)

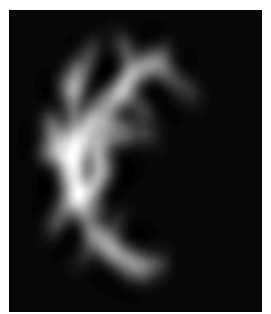

(e)

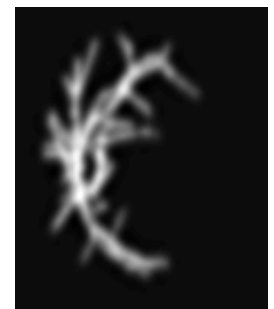

(f)

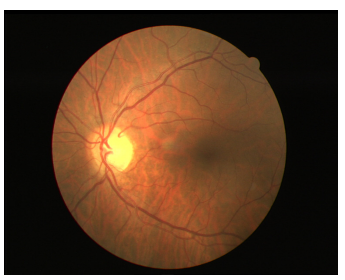

(b)

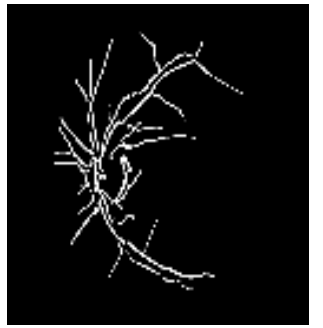

(d)

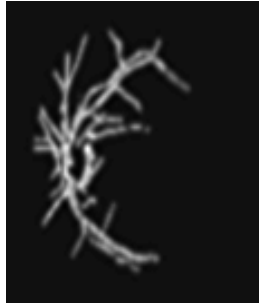

$(\mathrm{g})$

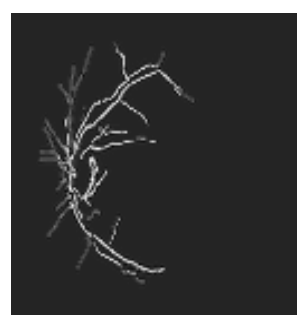

(h)

FigURE 2. Registration approach result for two interpolated vascular networks of the same individual, using a grid with $128 \times 128$ for the domain $\Omega$. Original retinal fundus images of the reference $R$ (a) and of the template $T$ (b). Vascular networks of $R$ (c), and of $T(\mathrm{~d})$. The results for the final transformed template vasculature $T(u)$ for a single scale $\theta=100$ (e), for two scales $\theta=\left[\begin{array}{ll}100 & 10\end{array}\right]$ (f), for three scales $\theta=\left[\begin{array}{lll}100 & 10 & 1\end{array}\right]$ (g) and finally for four scales $\theta=\left[\begin{array}{llll}100 & 10 & 1 & 0\end{array}\right]$ (h). In this example the elasticity coefficient $\mu=9$, and the values of the decision functions for the four scales in (h) are $d_{1}=0.7254, d_{2}=0.7475, d_{3}=0.6850$.

3.4. Decision identification function. Once the registration process has been finished, a decision function is necessary to determine the quality of the 


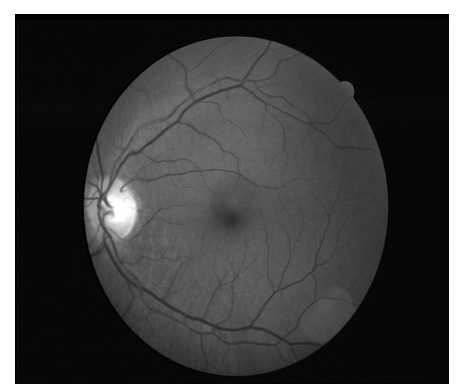

(a)

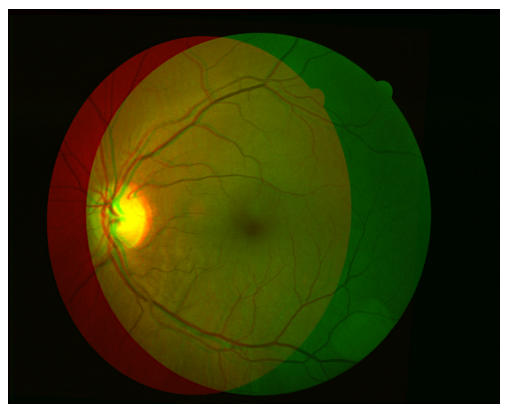

(c)

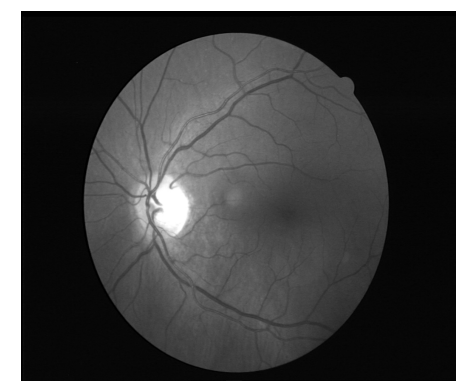

(b)

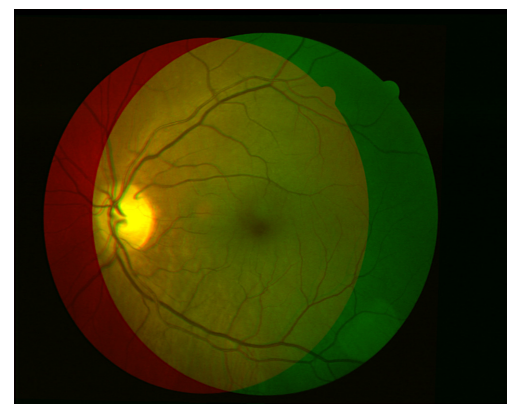

(d)

FiguRE 3. First row: Original green channels of the two left eye retinal fundus images of the same individual, displayed in the first row of Figure 2. (a) and (b) correspond to the reference $R$ and template $T$ images, respectively. Second row: overlapping of the registration result for these original green channels, by using the numerical solution $u$ of the proposed registration approach, generated when the vascular networks are interpolated with a $128 \times 128$ discretization in (c) and with a 256x256 discretization in (d) ( $\mu=9$ in both cases). The transformed template $T(u)$ corresponds to the red color, the reference $R$ to the green color, and the overlapping region to the yellow color. As expected a better fitting of the two vessel networks (reference and transformed template) can be observed in (d), with the finer discretization.

register process, to estimate how accurate the proposed registration approach is, and consequently to decide whether the pair of images correspond to the same subject eye, or not. In effect, the validation of a registration method is a central, but not solved problem, and we refer to [22] for a review of some existing methods. 


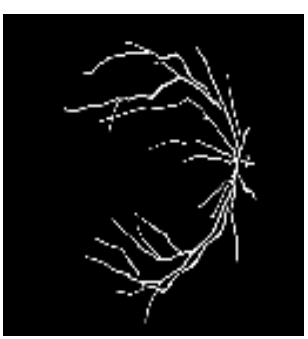

(a)

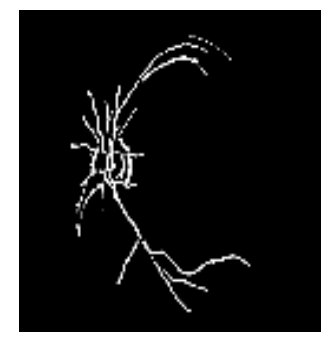

(d)

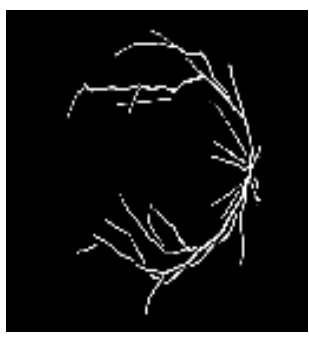

(b)

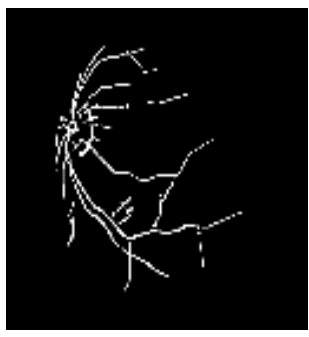

(e)

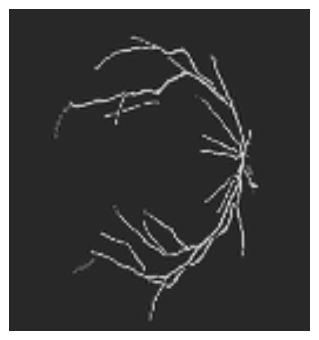

(c)

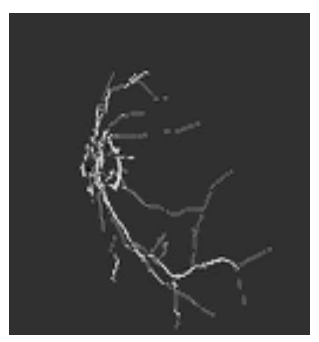

(f)

FiguRE 4. Registration results using the multiscale elastic image registration (four scales $\theta=\left[\begin{array}{llll}100 & 10 & 1 & 0\end{array}\right]$ ). First row: reference $R$ (a) and template $T$ (b) for the right eye of the same individual and final template $T(u)$ (c); the values of the decision functions are $d_{1}=0.4623, d_{2}=0.4660, d_{3}=0.4314$ Second row: reference $R$ (d) and template $T$ (e) for the left eyes of two different individuals and final template $T(u)(\mathrm{f})$; the values of the decision functions are $d_{1}=0.8443, d_{2}=1.0524, d_{3}=0.7630$.

We have tried several possible functions, as for instance, the normalized cross-correlation coefficient. But the best results (for a large dataset used in Section 4) were achieved, using a simple thresholding approach, with the following normalized functions

$$
\begin{gathered}
d_{1}:=\frac{\|\nabla T(u)-\nabla R\|_{L^{2}(\Omega)}}{\|\nabla R\|_{L^{2}(\Omega)}}, \quad d_{2}:=\frac{\|\nabla T(u)-\nabla R\|_{L^{1}(\Omega)}}{\|\nabla R\|_{L^{1}(\Omega)}}, \\
d_{3}:=\frac{\|T(u)-R\|_{L^{2}(\Omega)}}{\|R\|_{L^{2}(\Omega)}} .
\end{gathered}
$$


Here $u$ denotes the final numerical solution of the registration process, and $L^{2}(\Omega)$ and $L^{1}(\Omega)$ denote the space of square-integrable and integrable functions, respectively, in $\Omega$. We observe that the functions $d_{1}$ and $d_{2}$, defined in (7), quantify the similarity between the gradients of the reference and transformed template images in the norms of $L^{2}(\Omega)$ and $L^{1}(\Omega)$, respectively (these are also normalized by the norms, in $L^{2}(\Omega)$ and $L^{1}(\Omega)$, of the gradient of the reference images), whereas $d_{3}$ measures the similarity between the reference and transformed template images in the norm of $L^{2}(\Omega)$ (that is also normalized by the $L^{2}(\Omega)$ norm of the reference image).

Clearly, the smaller $d_{i}, i=1,2,3$, is, more likely is the image pair to belong to the same individual eye. Consequently the identification procedure is considered positive, meaning that the image pair belongs to the same individual eye, if $d_{i}$ is less or equal than a predefined threshold value $t h r_{i}$ (see Section 4). In the captions of Figures 2 and 4 it is indicated the values of $d_{i}$, for the pairs of the same and different subject eyes exhibited.

3.5. Summary of the algorithm. Below we summarize the complete identification algorithm, proposed in this paper, for a single pair composed of the reference and template retinal fundus images. The algorithm accepts this pair as an input and gives the binary decision whether the pair does or does not belong to the same individual eye.

\section{Algorithm}

(1) Input pair $(R, T)$ : reference and template images.

(2) Extraction of the green channels of $R, T$ denoted by $R_{g}$ and $T_{g}$. Resizing of these latter images to the size $1024 \times 1024=2^{9} \times 2^{9}$ (the resizing, with even number is needed for the next step, which uses curvelets).

(3) Denoising of $R_{g}$ and $T_{g}$ by applying fast discrete curvelet transform via wrapping. The denoised images are denoted by $R_{g}^{d}$ and $T_{g}^{d}$ (the superscript letter " $d "$ symbolizes denoised).

(4) Vasculature network extraction from $R_{g}^{d}$ and $T_{g}^{d}$, using wavelets, as explained in Section 3.1. The corresponding binary images are denoted by $R_{v}$ and $T_{v}$ (the subscript letter " $v$ " symbolizes vessels).

(5) Multiscale elastic image registration of the pair of binary images $\left(R_{v}, T_{v}\right)$ : 
(a) A fixed discretization of the domain $\Omega$, with $128 \times 128=2^{7} \times 2^{7}$ is considered, for both images (the goal is to speed up the numerical optimization, but finer grids can be used, and we refer to Figure 3 for an example with a finer grid).

(b) An affine pre-registration is performed at scale $\theta=100$, i.e. for the interpolated input image pair $\left(R_{v}(\theta), T_{v}(\theta)\right)$ (see Sections 3.2 and 3.3). Denoting by $u_{p r}$ its numerical solution, then the transformed template input for the next step is $T_{v}\left(u_{p r}\right)$. This means in the next step the input pair is $\left(R_{v}, T_{v}\left(u_{p r}\right)\right)$.

(c) A loop over four scales of $\theta[\theta=100,10,1,0]$ is considered for carrying out the multiscale elastic optimization. At scale $\theta$, the interpolated input image pair is $\left(R_{v}(\theta), T_{v}\left(u_{p r}\right)(\theta)\right)$ (following the notations of Section 3.3), and the numerical minimizer $u_{\theta}$ is the starting point for the elastic registration of the next finer scale.

For each scale a Gauss-Newton like method is used for solving the optimization problem (5).

The values for the Lamé constants $\mu, \lambda$, that depend on the material (in this case the vessels), and the value for the regularizing parameter $\alpha$ are the same for all the scales $(\mu=9, \lambda=0$, $\alpha=800)$.

(6) Finally, the decision function $d_{1}$, defined in (7), is applied : if $d_{1} \leq t h r_{1}$ (where $t h r_{1}$ is a predefined threshold) the identification is positive, i.e. the input pair of images correspond to the same individual eye, otherwise the pair is considered negative and the two images belong to different individuals.

\section{Results}

We now evaluate the performance of our algorithm using standard performance measures such as sensitivity, specificity and accuracy. The algorithm is implemented in MATLAB R2012a on a iMac computer running OS X 10.9.1 with $2.93 \mathrm{GHz}$ Intel Core 2 Duo processor and 4 GB of RAM. Our testing data set includes 946 images from 339 different subjects. Of these, 230 images from 58 different subjects correspond to the patients followed in age-related macular degeneration (AMD) disease, 281 images from 81 different subjects correspond to the patients followed in diabetic retinopathy (DR) disease, and 435 images from 200 different subjects correspond to the healthy patients. From these total 946 images we formed a data set having 2567 true 


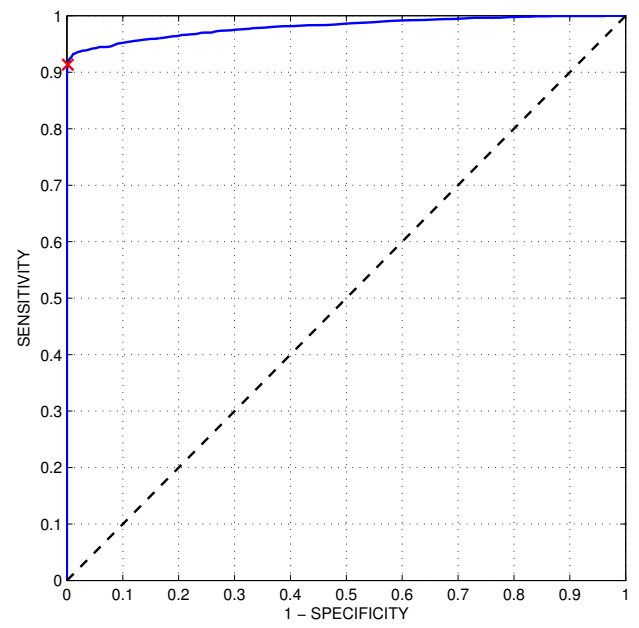

(a)

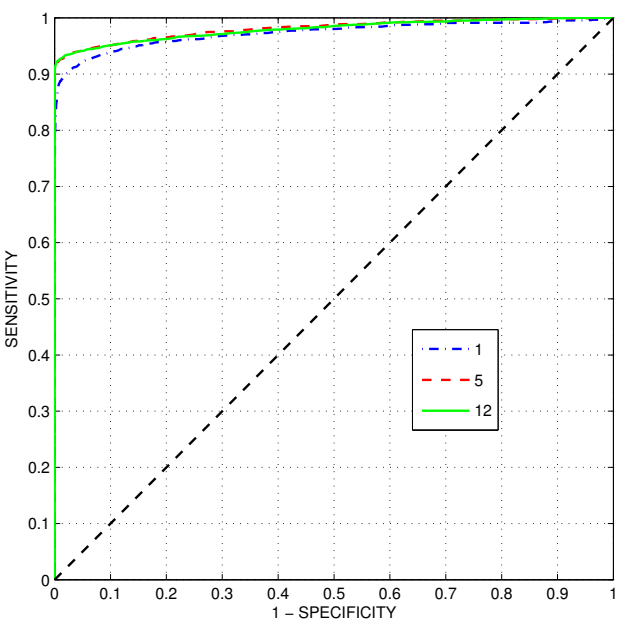

(b)

FiguRE 5. Left: ROC curve for the decision identification function $d_{1}$ with $\mu=9$, the red cross mark indicates the point corresponding to $t h r_{1}=0.8178$. Right: ROC curves for the decision identification function $d_{1}$ with $\mu=1,5,12$.

pairs (i.e. in each pair both the images belong to the same subject) and 19113 false pairs (i.e. in each pair the images belong to different subjects). The images are provided by the company Retmarker (http://www.retmarker.com/), and are taken during an ongoing screening program in Portugal.

To capture the statistical nature of the testing, we consider the receiver operator characteristic (ROC) curves [8]. In our case, the ROC curve considered is the graphical plot of the sensitivity versus the specificity, for the decision identification function $d_{1}$ defined in Section 3.4. We have generated the ROC curve by varying the threshold value and an optimal threshold is selected as one that maximizes the accuracy. The threshold is a numeric value defined by the function $d_{1}$, that produces a binary classifier: if for a pair of images the value of the decision identification function $d_{1}$ is below the threshold, the classifier produces a positive result $(\mathrm{P})$, else a negative $(\mathrm{N})$ result. If the pair of images belongs to the class of true pairs and it is classified as negative, it is counted as a false negative (FN); if it is classified as positive, it is counted as a true positive (TP). If the pair of images belongs to the class of false pairs and it is classified as positive, it is counted as a false positive (FP); if it is classified as negative, it is counted as a true negative 
(TN). Then the sensitivity and specificity are defined as follows

$$
\begin{aligned}
& \text { sensitivity }=\frac{\text { number of TP }}{\text { number of } \mathrm{TP}+\text { number of } \mathrm{FN}} \\
& \text { specificity }=\frac{\text { number of } \mathrm{TN}}{\text { number of } \mathrm{TN}+\text { number of } \mathrm{FP}} .
\end{aligned}
$$

Sensitivity represents the ability of the algorithm to correctly classify a pair of images as true pairs, while specificity represents the ability of the algorithm to correctly classify a pair of images as false pairs. Obviously, one would like both the sensitivity and the specificity to be as high as possible. However, there is always a trade off between the two. To visually represent this trade off we use the ROC curve. If the classifier makes a decision randomly with equal probability, then the ROC curve will be along the diagonal line. In addition, a ROC curve above the diagonal represents good classification results, and a ROC curve below the diagonal represents bad classification results. The ROC curves showing the relationship between sensitivity and specificity are shown in Figure 5, respectively, for $\mu=9$ and $\mu=1,5,12$.

The overall performance of the algorithm is measured in terms of the accuracy, as defined below

$$
\text { accuracy }=\frac{\text { number of } \mathrm{TN}+\text { number of TP }}{\text { number of } \mathrm{TN}+\text { number of } \mathrm{FP}+\text { number of } \mathrm{TP}+\text { number of } \mathrm{FN}} \text {. }
$$

We tested the algorithm for different values of $\mu$ and observed that the best results are obtained with $\mu=9$. Note that the results for $\mu=9$ are marginally better than the results for $\mu=5,12$, as we can also see from Figure 5 that the ROC curves for $\mu=5,9,12$ are very similar. Using $\mu=9$, the values of specificity, sensitivity, and accuracy are 99.998\%, 91.350\%, and $98.830 \%$, respectively, for the threshold 0.8178 . Thus, the proposed algorithm is highly promising to be used in identifying individuals, and hence in biometric applications.

\section{Conclusions}

Identifying individuals based on retinal images is one of the popular biometrics approaches. To this end we have proposed an identification method based on retinal fundus images. It consists in the extraction of the retina vasculatures, from the images, and their subsequent registration using a multiscale elastic registration approach. For deciding whether or not the pair of images belong to a particular individual, a suitable decision identification function is introduced. The experimental results on a large data set show the extreme efficacy of this method. 
In a recent work [9] we have proposed a pattern classification of retinal fundus images that relies on the computation of appropriate function norms of the corresponding retinal vessel network images. It is our intention, as future work, to test in a large dataset, the combination of this pattern classification, as a pre-identification step, with the retina identification strategy (proposed in this paper) as a second identification step. The goal is to reduce substantially the search and speed up the global identification procedure.

\section{Acknowledgment}

This work was partially supported by the project PTDC/MATNAN/0593/2012, and also by CMUC (Center for Mathematics, University of Coimbra) and FCT (Portugal), through European program COMPETE/ FEDER and project PEst-C/MAT/UI0324/2013. The authors would also like to thank Dr. Gonçalo Quadros for having suggested the study of the interesting and challenging retina topic.

\section{References}

[1] M. D. Amiri, F. A. Tab, and W. Barkhoda. Retina identification based on the pattern of blood vessels using angular and radial partitioning. In J. Blanc-Talon, W. Philips, D. Popescu, and P. Scheunders, editors, Advanced Concepts for Intelligent Vision Systems, volume 5807 of Lecture Notes in Computer Science, pages 732-739. Springer Berlin Heidelberg, 2009.

[2] P. Bankhead, C. N. Scholfield, J. G. McGeown, and T.M. Curtis. Fast retinal vessel detection and measurement using wavelets and edge location refinement. PloS one, 7:e32435, 2012.

[3] R. Bolle and S. Pankanti. Biometrics, Personal Identification in Networked Society: Personal Identification in Networked Society. Kluwer Academic Publishers, Norwell, MA, USA, 1998.

[4] C. Broit. Optimal registration of deformed images. Ph.D. Thesis, Computer an Information Science, University of Pensylvania, 1981.

[5] E. Candes, L. Demanet, D. Donoho, and L. Ying. Fast discrete curvelet transforms. Multiscale Modeling E Simulation, 5:861-899, 2006.

[6] A. Dehghani, Z. Ghassabi, H. A. Moghddam, and M. Moin. Human recognition based on retinal images and using new similarity function. EURASIP Journal on Image and Video Processing, 2013, 2013.

[7] H. Farzin, H. Abrishami-Moghaddam, and M.-S. Moin. A novel retinal identification system. EURASIP J. Adv. Signal Process, 2008:280635, 2008.

[8] T. Fawcett. An introduction to ROC analysis. Pattern Recogn. Lett., 27:861-874, 2006.

[9] I. N. Figueiredo, J. S. Neves, S. Moura, C. M. Oliveira, and J.D. Ramos. Pattern classes in retinal fundus images based on function norms. In Computational Modeling of Objects Presented in Images. Fundamentals, Methods, and Applications, pages 95-105. Lecture Notes in Computer Science, Vol. 8641, Springer International Publishing Switzerland, 2014. 
[10] E. Haber, U. M Ascher, and D. Oldenburg. On optimization techniques for solving nonlinear inverse problems. Inverse problems, 16(5):1263, 2000.

[11] E. Haber and J. Modersitzki. A multilevel method for image registration. SIAM Journal on Scientific Computing, 27(5):1594-1607, 2006.

[12] R. B. Hill. Retina identification. In A. K. Jain, R. Bolle, and S. Pankanti, editors, Biometrics, pages 123-141. Springer US, 1996.

[13] X. Meng, Y. Yin, G. Yang, and X. Xi. Retinal identification based on an improved circular Gabor filter and scale invariant feature transform. Sensors, 13:9248-9266, 2013.

[14] J. Modersitzki. Numerical methods for image registration. OUP Oxford, 2003.

[15] J. Modersitzki. FAIR: flexible algorithms for image registration, volume 6. SIAM, 2009.

[16] M. Ortega, C. Mariño, M. G. Penedo, M. Blanco, and F. González. Biometric authentication using digital retinal images. In Proceedings of the 5th WSEAS International Conference on Applied Computer Science, ACOS'06, pages 422-427, Stevens Point, Wisconsin, USA, 2006.

[17] M. Ortega, M. G. Penedo, J. Rouco, N. Barreira, and M. J. Carreira. Retinal verification using a feature points-based biometric pattern. EURASIP J. Adv. Signal Process, 2009:2:1$2: 13,2009$.

[18] M. Sabaghi, S. Hadianamrei, A. Zahedi, and M. Lahiji. A new partitioning method in frequency analysis of the retinal images for human identification. Journal of Signal and Information Processing, 2:274-278, 2011.

[19] C. Simon and I. Goldstein. A new scientific method of identification. N. Y. State J. Med, 35:901-906, 1935.

[20] J.-L. Starck, J. Fadili, and F. Murtagh. The undecimated wavelet decomposition and its reconstruction. IEEE Transactions on Image Processing, 16:297-309, 2007.

[21] P. Tower. The fundus oculi in monozygotic twins: Report of six pairs of identical twins. A.M.A. Archives of Ophthalmology, 54:225-239, 1955.

[22] B. Zitova and J. Flusser. Image registration methods: a survey. Image and vision computing, 21(11):977-1000, 2003.

ISABel NARra Figueiredo

CmuC, Department of Mathematics, University of Coimbra, Portugal.

E-mail address: isabelf@matuc.pt

$U R L:$ http://www.mat.uc.pt/ isabelf

SUNIL Kumar

CMUC, Department of Mathematics, University of Coimbra, Portugal.

E-mail address: skumar@mat.uc.pt

JÚLIO S. NEVES

CMUC, Department of Mathematics, University of Coimbra, Portugal.

E-mail address: jsn@mat.uc.pt

$U R L:$ http://www.mat.uc.pt/ jsn

Susana Moura

CmuC, Department of Mathematics, University of Coimbra, Portugal.

E-mail address: smpsd@mat.uc.pt

$U R L:$ http://www.mat.uc.pt/ $\sim \mathrm{smpsd}$

Carlos Manta Oliveira

Universidade Nova de Lisboa, Portugal and Retmarker 
20 I.N. FIGUEIREDO, S. KUMAR, J.S. NEVES, S. MOURA, C.M. OLIVEIRA AND J.D. RAMOS

E-mail address: carlos.a.oliveira@critical-health.com

URL: http://www.retmarker.com/

JoÃo Diogo RAMOS

RETMARKER

E-mail address: jramos@critical-health.com

URL: http://www.retmarker.com/ 